\title{
BLADDER DISEASE, Part A
}

Research Concepts and

Clinical Applications 


\section{ADVANCES IN EXPERIMENTAL MEDICINE AND BIOLOGY}

Editorial Board:

NATHAN BACK, State University of New York at Buffalo

IRUN R. COHEN, The Weizmann Institute of Science

DAVID KRITCHEVSKY, Wistar Institute

ABEL LAJTHA, N. S. Kline Institute for Psychiatric Research

RODOLFO PAOLETTI, University of Milan

Recent Volumes in this Series

Volume 533

RETINAL DEGENERATIONS: Mechanisms and Experimental Theory

Edited by Matthew M. LaVail, Joe G. Hollyfield, and Robert E. Anderson

Volume 534

TISSUE ENGINEERING, STEM CELLS, AND GENE THERAPIES

Edited by Y. Murat Elçin

Volume 535

GLYCOBIOLOGY AND MEDICINE

Edited by John S. Axford

Volume 536

CHEMORECEPTION: From Cellular Signaling to Functional Plasticity

Edited by Jean-Marc Pequignot, Constancio Gonzalez, Colin A. Nurse,

Nanduri R. Prabhakar, and Yvette Dalmaz

Volume 537

MATHEMATICAL MODELING IN NUTRITION AND THE HEALTH SCIENCES

Edited by Janet A. Novotny, Michael H. Green, and Ray C. Boston

Volume 538

MOLECULAR AND CELLULAR ASPECTS OF MUSCLE CONTRACTION

Edited by Haruo Sugi

Volume 539

BLADDER DISEASE, Part A and Part B: Research Concepts and Clinical Applications

Edited by Anthony Atala and Debra Slade

Volume 540

OXYGEN TRANSPORT TO TISSUE, VOLUME XXV

Edited by Maureen S. Thorniley, David K. Harrison, and Philip E. James

Volume 541

FRONTIERS IN CLINICAL NEUROSCIENCE: Neurodegeneration and Neuroprotection Edited by László Vécsei

Volume 542

QUALITY OF FRESH AND PROCESSED FOODS

Edited by Fereidoon Shahidi, Arthur M. Spanier, Chi-Tang Ho, and Terry Braggins

A Continuation Order Plan is available for this series. A continuation order will bring delivery of each new volume immediately upon publication. Volumes are billed only upon actual shipment. For further information please contact the publisher. 


\section{BLADDER DISEASE, Part A \\ Research Concepts and \\ Clinical Applications}

Edited by

Anthony Atala

Children's Hospital and Harvard Medical School

Boston, Massachusetts

and

Debra Slade

Ridgefield, Connecticut

Springer Science+Business Media, LLC 


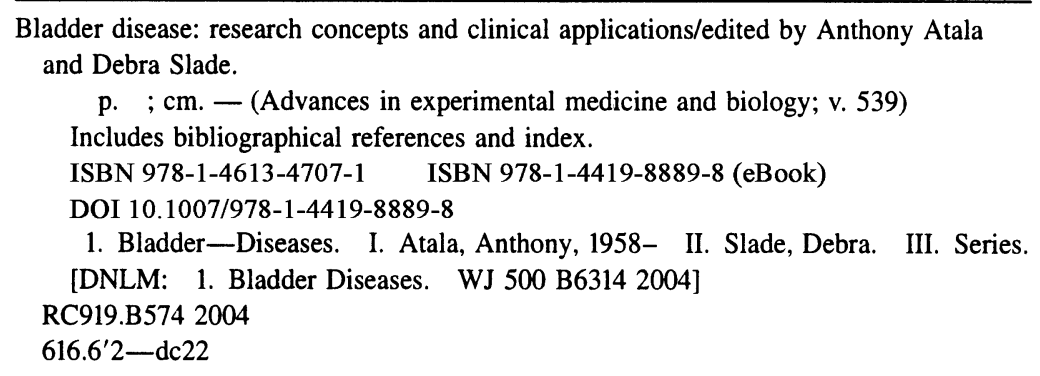

\section{ISSN 0065-2598}

ISBN 978-1-4613-4707-1

C2003 Springer Science+Business Media New York

Originally published by Kluwer Academic/Plenum Publishers, New York in 2003

Softcover reprint of the hardcover 1st edition 2003

All rights reserved

No part of this book may be reproduced, stored in a retrieval system, or transmitted in any form or by any means, electronic, mechanical, photocopying, microfilming, recording, or otherwise, without written permission from the Publisher, with the exception of any material supplied specifically for the purpose of being entered and executed on a computer system, for exclusive use by the purchaser of the work.

Permissions for books published in Europe: permissions@wkap.nl Permissions for books published in the United States of America: permissions@wkap.com 
This book is dedicated to:

Katherine, Christopher, and Zachary (AA) and

Peter, Laura, and Emma (DS).

We would also like to express our sincere gratitude to Ti Klingler-Desai for her editorial coordination and final preparation of the manuscript. 


\section{CONTRIBUTORS}

N. Scott Adzick

Children's Hospital of Philadelphia

Division of Pediatric Surgery

Philadelphia, PA 19104

Karl-Erik Andersson

Albert Einstein College of

Medicine

Department of Clinical

Pharmacology

Bronx, NY 10460

Peter Ardelt

Medical University of Lübeck

Department of Urology

23538 Lübeck, Germany

Anthony Atala

Children's Hospital Boston

Department of Urology

Boston, MA 02115

Kazem M. Azadzoi

Boston University School of

Medicine

Boston, MA 02130

W. Ripley Ballou

MedImmune, Inc.

Gaithersburg, MD 20878

Sudip K. Bandyopadhyay

The Cleveland Clinic Foundation

Lerner Research Institute

Cleveland, $\mathrm{OH} 44195$

James A. Bassuk

Children's Hospital and Regional

Medical Center

Division of Pediatric Urology

Seattle, WA 98105
Matt Beckman

University of Wisconsin School of

Medicine

Division of Urology

Madison, WI 53706

Gilbert R. Besson

Hôpital Jean Rostand

Division of Rehabilitation

Ivry-sur-Seine, France

Charles D. Best

Stanford University Medical Center

Department of Urology

Palo Alto, CA 94305

Karl-Horst Bichler

University of Tübingen

Department of Urology

Tübingen, Germany

Linda M. Birnby

Stratton VA Medical Center

Albany Medical College

Albany, NY 12208

Dale E. Bjorling

University of Wisconsin School of

Veterinary Medicine

Department of Surgical Sciences

Madison, WI 53706

Andreas Böhle

Medical University of Lübeck

Department of Urology

Lübeck, Germany

Adrian D. Bonev

University of Virginia College of

Medicine

Department of Pharmacology

Burlington, VT 05405 
William Boucher

Tufts University School of

Medicine

Department of Pharmacology and

Experimental Therapeutics

Boston, MA 02111

Alison F. Brading

Oxford Continence Group

Department of Pharmacology

Oxford, United Kingdom

Olaf A. Brinkmann

University of Muenster

Muenster, Germany

\section{J. L. Brod}

Friedrich Alexander University of

Erlangen Nuremberg

Department of Urology

Erlangen, Germany

G. Brodner

University of Muenster

Muenster, Germany

Douglas A. Canning

Children's Hospital of Philadelphia

Division of Pediatric Urology

Philadelphia, PA 19104

Thurston Carleton

The George Washington

University School of Medicine \&

Medical Center

Washington DC 20037

Michael Carr

Children's Hospital of Philadelphia

Division of Urology

Philadelphia, PA 19104
Bulent Cetinel

University Cerrahpasa School of

Medicine

Department of Urology

Istanbul, Turkey

Samuel K. Chacko

Children's Hospital of Philadelphia

Division of Urology

Philadelphia, PA 19104

Brahim Chaqour

University of Pennsylvania School

of Dental Medicine

Philadelphia, PA 19104

George Chiang

Tufts University School of

Medicine

Department of Pharmacology and

Experimental Therapeutics

Boston, MA 02111

Jong M. Choe

University of Cincinnati Medical

Center

Division of Urology

Cincinnati, OH 45267

George J. Christ

Albert Einstein College of Medicine

Bronx, NY 10461

Kimberly Cochrane

Children's Hospital and Regional

Medical Center

Seattle, WA

Timothy Crombleholme

Children's Hospital of Philadelphia

Division of Pediatric Surgery

Philadelphia, PA 19104 
Margot Damaser

Loyola University of Chicago

School of Medicine

Maywood, IL 60153

Nancy S. Day

Albert Einstein College of

Medicine

Bronx, NY 10461

Larry A. Demco

Rockyview General Hospital

Calgary, AB, Canada

J. Dörsam

Upstate Medical University

Department of Pathology

Syracuse, NY 13210

Karyn S. Eilber

University of California, Los

Angeles School of Medicine

Department of Urology

Los Angeles, CA 90095

Osama M. Elashry

Tanta University Hospital

Department of Urology

Tanta, Egypt

A. Elbadawi

Upstate Medical University

Department of Pathology

Syracuse, NY 13210

Ada Elgavish

University of Alabama

Division of Urology

Birmingham, AL 35294

Ahmed S. Elmahouky

Tanta University Hospital

Department of Urology

Tanta, Egypt
Mahmoud A. Emran

Tanta University Hospital

Department of Urology

Tanta, Egypt

Gerhard Feil

University of Tübingen

Department of Urology

Tübingen, Germany

Alan Flake

Children's Hospital of Philadelphia

Division of Pediatric Surgery

Philadelphia, PA 19104

Peter Frey

Centre Hospitalier Universitaire

Vaudois Lausanne, Switzerland

Roger P. Goldberg

Evanston Northwestern Hospital

Evanston Continence Center

Evanston, IL 60201

ChaoLiang Gong

Children's Hospital of Philadelphia

Division of Urology

Philadelphia, PA 19104

Tobias Götz

University of Tübingen

Department of Urology

Tübingen, Germany

Marlon Green

Tufts University School of

Medicine

Department of Pharmacology and

Experimental Therapeutics

Boston, MA 02111

H. Barton Grossman

University of Texas

M.D. Anderson Cancer Center

Houston, TX 77030 
A. Haferkamp

Upstate Medical University

Department of Pathology

Syracuse, NY 13210

James Hahn

The George Washington

University School of Medicine \&

Medical Center

Washington DC 20037

T. Hanai

Kinki University School of

Medicine

Osaka, Japan

Moritz Hansen

VA Palo Alto Health Care System

Section of Urology

Palo Alto, CA 94304

Martha A. Hass

Albany College of Pharmacy

Department of Basic and

Pharmaceutical Sciences

Albany, NY 12208

Thomas J. Heppner

University of Vermont College of

Medicine

Department of Pharmacology

Burlington, VT 05405

Gerald M. Herrera

University of Vermont College of

Medicine

Department of Pharmacology

Burlington, VT 05405

Lothar Hertle

University of Muenster

Department of Urology

Muenster, Germany
Ralf Herwig

University of Muenster

Muenster, Germany

David Hill-Eubanks

University of Vermont College of

Medicine

Department of Pharmacology

Burlington, VT 05405

Pamela S. Howard

University of Pennsylvania School

of Dental Medicine

Children's Hospital of Philadelphia

Division of Pediatric Urology

Philadelphia, PA 19104

Xing Cheng Hua

The George Washington University

School of Medicine \& Medical

Center

Washington DC 20037

Ann. M. Hubbard

Children's Hospital of Philadelphia

Departments of Surgery and

Radiology

Philadelphia, PA 19104

Joel C. Hutcheson

Children's Hospital of Philadelphia

Division of Urology

Philadelphia, PA 19104

Mitsuhiko Inaba

Kyoto Prefectural University of

Medicine

Kyoto, Japan

Takahiro Isono

Shiga University of Medical

Science

Central Research Laboratory

Otsu, Japan 
B. Ittah

Capital Health Region

Department of Urology

Victoria, BC, Canada

Tsuyoshi Iwata

Kyoto Prefectural University of

Medicine

Department of Urology

Kyoto, Japan

Gabriella Janni

University of California, San

Francisco

Department of Neurological

Surgery

San Francisco, CA 94143

Luc Jasmin

University of California, San

Francisco

Department of Neurological

Surgery

San Francisco, CA 94143

Sheila Jeudy

Tufts University School of

Medicine

Department of Pharmacology and

Experimental Therapeutics

Boston, MA 02111

Roger Kaufman

The George Washington

University School of Medicine \&

Medical Center

Washington DC 20037

Ingo Kausch

Medical University of Lübeck

Department of Urology

Lübeck, Germany
Fernando J. Kim

Loyola University of Chicago

School of Medicine

Maywood, IL 60153

Andrew J. Kirsch

Children's Hospital of Philadelphia

Division of Pediatric Urology

Philadelphia, PA 19104

Munekado Kojima

Kyoto Prefectural University of

Medicine

Department of Urology

Kyoto, Japan

Lev Krasnopolsky

Children's Hospital of Philadelphia

Division of Urology

Philadelphia, PA 19104

F. Steffen Krause

University of Tübingen

Department of Urology

Tübingen, Germany

R. Kühn

Friedrich Alexander University of

Erlangen Nuremberg

Department of Urology

Erlangen, Germany

T. Kurita

Kinki University School of

Medicine

Department of Urology

Osaka, Japan

Sven Lahme

University of Tübingen

Department of Urology

Tübingen, Germany 
Raymond S. Lance

University of Texas

M.D. Anderson Cancer Center

Houston, TX 77030

Solomon Langermann

MedImmune, Inc.

Gaithersburg, MD 20878

Jim Moo Lee

Yonsei University College of

Medicine

Department of Urology

South Korea

Young Tae Lee

Pochon CHA University

South Korea

Richard Letourneau

Tufts University School of

Medicine

Department of Pharmacology and

Experimental Therapeutics

Boston, MA 02111

Robert M. Levin

Albany College of Pharmacy

Department of Basic and

Pharmaceutical Sciences

Albany, NY 12208

Louis S. Liou

The Cleveland Clinic Foundation

Lerner Research Institute

Cleveland, $\mathrm{OH} 44195$

I. Jenna Liu

Section of Urology

VA Palo Alto Health Care System

Palo Alto, CA 94304
Edward J. Macarak

University of Pennsylvania School

of Dental Medicine

Children's Hospital of Philadelphia

Division of Pediatric Urology

Philadelphia, PA 19104

Michael J. Manyak

The George Washington University

School of Medicine \& Medical

Center

Washington DC 20037

Surena F. Matin

The Cleveland Clinic Foundation

Lerner Research Institute

Cleveland, $\mathrm{OH} 44195$

S. Matsumoto

Kinki University School of

Medicine

Sakai Hospital

Osaka, Japan

Marelyn Medina

Rio Grande Regional Hospital

McAllen, TX 78503

Tsuneharu Miki

Kyoto Prefectural University of

Medicine

Department of Urology

Kyoto, Japan

Wendy L. Milonovich

Albany Medical College

Stratton VA Medical Center

Albany, NY 12208

Gina M. Minetti

Loyola University of Chicago

School of Medicine

Maywood, IL 60153 
Michael E. Mitchell

Children's Hospital and Regional

Medical Center

Division of Pediatric Urology

Seattle, WA 98105

Amithi Narendran

Albany Medical College

Stratton VA Medical Center

Albany, NY 12208

H. J. Nelde

University of Tübingen

Department of Urology

Tübingen, Germany

Mark T. Nelson

University of Vermont College of

Medicine

Department of Pharmacology

Burlington, VT 05405

Pierre P. Nelson

Hôpital Jean Rostand

Division of Rehabilitation

Ivry-sur-Seine, France

Endre Neulander

University of Florida

Division of Urology

Gainesville, FL 32601

Milomir Ninkovic

University of Innsbruck Medical

School

Department of Plastic and

Reconstructive Surgery

Innsbruck, Austria

Matilde Nino-Murcia

Stanford University Medical

Center

Department of Urology

Stanford, CA 94305
Ichiro Nishimura

University of California, Los

Angeles School of Medicine

Department of Urology

Los Angeles, CA 90095

N. Ohnishi

Kinki University School of

Medicine

Department of Urology

Osaka, Japan

Yusaku Okada

Shiga University of Medical

Science

Department of Urology

Otsu, Japan

Dong Soo Park

Pochon CHA University

Department of Urology

South Korea

C. Lowell Parsons

University of California, San Diego

Medical Center

Division of Urology

San Diego, CA 92103

Phani Patra

Tufts University School of

Medicine

Department of Pharmacology and

Experimental Therapeutics

Boston, MA 02111

Katerina Persson

Albert Einstein College of Medicine

Bronx, NY 10461

Patricia G. Phillips

Albany Medical College

Stratton VA Medical Center

Albany, NY 12208 
Theresa Quinn

Children's Hospital of Philadelphia

Division of Pediatric Urology

Philadelphia, PA 19104

Raymond R. Rackley

The Cleveland Clinic Foundation

Lerner Research Institute

Cleveland, $\mathrm{OH} 44195$

Shlomo Raz

University of California, Los

Angeles School of Medicine

Department of Urology

Los Angeles, CA 90095

Inoel Rivera

University of Florida

Division of Urology

Gainesville, FL 32601

Dorothea Rohrmann

Urologische Universitätsklinik der

RWTH

Aachen, Germany

Joel Rosenbloom

University of Pennsylvania School of Dental Medicine

Philadelphia, PA 19104

S. Rowan

Capital Health Region

Department of Urology

Victoria, BC, Canada

Ricardo Saban

University of Texas Medical

Branch

Departments of Internal Medicine

and Surgery

Galveston, TX 77555
Provash C. Sadhukhan

The Cleveland Clinic Foundation

Lerner Research Institute

Cleveland, $\mathrm{OH} 44195$

Peter K. Sand

Evanston Northwestern Center

Evanston Continence Center

Evanston, IL 60201

Grannum R. Sant

Tufts University School of

Medicine

Department of Pharmacology and

Experimental Therapeutics

Boston, MA 02111

Kristin Santangelo

The George Washington University

School of Medicine \& Medical

Center

Washington DC 20037

Cristian Santizo

Albert Einstein College of Medicine Bronx, NY 10461

W. Schafhauser

Friedrich Alexander University of

Erlangen Nuremberg

Department of Urology

Erlangen, Germany

Paul F. Schellhammer

Eastern Virginia Medical School and the Virginia Prostate Center Norfolk, VA 23510

K. M. Schrott

Friedrich Alexander University of

Erlangen Nuremberg

Department of Urology

Erlangen, Germany 
George M. Segall

VA Palo Alto Health Care System

Palo Alto, CA 94304

Ahmed Shafik

Cairo University

Department of Surgery and

Experimental Research

Cairo, Egypt

Karl-Dietrich Sievert

University of Muenster

Department of Urology

Muenster, Germany

Howard M. Snyder

Children's Hospital of Philadelphia

Division of Pediatric Urology

Philadelphia, PA 19104

Paul C. Stein

University of California, San

Diego Medical Center

Division of Urology

San Diego, CA 92103

Raimund Stein

Children's Hospital of Philadelphia

Division of Urology

Philadelphia, PA 19104

G. Steinhoff

Capital Health Region

Department of Urology

Victoria, BC, Canada

Arnulf Stenzl

University of Innsbruck Medical

School

Department of Urology

Innsbruck, Austria
Cortino Sukotjo

University of California, Los

Angeles School of Medicine

Department of Urology

Los Angeles, CA 90095

Stephanie Symes

Oxford Continence Group

Department of Pharmacology

Oxford, United Kingdom

Motoyoshi Tanaka

University of Texas

M.D. Anderson Cancer Center

Houston, TX 77030

Tsutomu Tanaka

Shiga University of Medical

Science

Department of Urology

Otsu, Japan

Martha K. Terris

Stanford University Medical Center

Department of Urology

Stanford, CA 94305

Theoharis C. Theoharides

Tufts University School of

Medicine

Department of Pharmacology and

Experimental Therapeutics

Boston, MA 02111

Osamu Ukimura

Kyoto Prefectural University of

Medicine

Department of Urology

Kyoto, Japan

Mira Valcic

Albert Einstein College of Medicine Bronx, NY 10461 
Françoise A. Valentini

Hôpital Jean Rostand

Division of Rehabilitation

Université Pierre et Marie Curie

Ivry-sur-Seine and Paris, France

Karicheti Venkateswarlu

Álbert Einstein College of

Medicine

Bronx, NY 10461

Antonia Vlahou

Eastern Virginia Medical School and the Virginia Prostate Center

Norfolk, VA 23510

Zev Wajsman

University of Florida

Division of Urology

Gainesville, FL 32601

Raymond J. Walsh

The George Washington

University School of Medicine \&

Medical Center

Washington DC 20037

Hong-Zhan Wang

Albert Einstein College of

Medicine

Bronx, NY 10461

Hans W. Wechsel

University of Tübingen

Department of Urology

Tübingen, Germany

Alan J. Wein

Children's Hospital of Philadelphia

Division of Urology

Philadelphia, PA 19104
Jeffrey P. Weiss

New York Hospital

Cornell Medical Center

New York, NY 10022

George L. Wright, Jr.

Eastern Virginia Medical School

and the Virginia Prostate Center

Norfolk, VA 23510

Tatsuhiro Yoshiki

Shiga University of Medical

Science

Department of Urology

Otsu, Japan

Takeshi Yuasa

Shiga University of Medical

Science

Department of Urology

Otsu, Japan

Stephen A. Zderic

Children's Hospital of Philadelphia

Division of Urology

Philadelphia, PA 19104

Jian Zhang

University of California, San Diego

Medical Center

San Diego, CA 92103

Yuan Yuan Zhang

Centre Hospitalier Universitaire

Vaudois Lausanne, Switzerland

Weixin Zhao

Albert Einstein College of Medicine

Bronx, NY 10461

Andreas Zumbraegel

University of Tübingen

Department of Urology

Tübingen, Germany 


\section{PREFACE}

In 1996, the National Bladder Foundation (NBF) was founded by a dedicated group of physicians and researchers propelled by the urgent need to find better treatments for bladder disease. Committed to increasing bladder disease research and to supporting its research community, the NBF coordinates and sponsors the International Bladder Symposium (IBS) in Washington, DC. Now considered to be a premier scientific assembly, the IBS brings together international leaders in bladder disease research to present and discuss their findings. It is the only international conference where all areas of bladder disease research are exclusively covered and where bladder disease researchers are provided with a unique opportunity to share their results and theories.

IBS participants contributed the research papers included in this publication in 2000 and 2001. All substantial areas of bladder disease research are addressed, including oncology and cellular biology, neurophysiology, neurogenic bladder and incontinence, immunology, inflammation and infection, muscle, matrix and obstruction, and new frontiers and therapies of the bladder. Assembled in one publication, these papers and their findings demonstrate the high scientific caliber of the dedicated researchers in this field and the potential for significant discoveries in treatment options in the next decade.

The National Bladder Foundation is very grateful to the researchers who have contributed to the International Bladder Symposium and to this publication. We are especially grateful to the tireless efforts of the bladder research community who continue to pursue their important work despite funding inequity and inadequate recognition. The NBF would like to acknowledge the support of Congressional representatives, particularly Senator Harry Reid of Nevada, for helping to highlight the need for more bladder disease research in their yearly appropriations reports. The NBF is also grateful to Allen Spiegel, Josephine Briggs, Stuart Howards, and Leroy Nyberg of the National Institute of Diabetes, Digestive, and Kidney Diseases for their support of bladder disease research and for their recent efforts to address the funding inequities in their Bladder Research Progress 
Review Group (PRG) Report, chaired by Linda Shortliffe and Monica Liebert. Finally, we thank the sponsors of the International Bladder Symposium and our dedicated partners in industry who are also working to find better treatments and cures for bladder disease.

Bladder disease is a major health crisis for America. Afflicting over 35 million people, the cost to this country is enormous and grows as the population ages. Most of the major diseases lack adequate treatment options resulting in frustration and anguish for the patient and caregiver. Many patients continue to suffer in silence because of the still ongoing stigma associated with their afflictions and do not seek what treatments are available. This book is dedicated to the worldwide bladder community, its patients and their caregivers and to all who care passionately about bladder disease research.

Anthony Atala, M.D.

Debra K.A. Slade, MA (Oxon)

Editors 


\section{National Bladder Foundation Board of Directors}

Anthony Atala, M.D.

Harvard Medical School

Boston, MA

Michael J. Droller, M.D.

Mount Sinai Medical Center

New York, NY

Monica Liebert, Ph.D.

AUA Research Director

Baltimore, MD

John McConnell, M.D.

University of Southwestern

Medical Center

Dallas, TX

Timothy L. Ratliff, Ph.D.

University of Iowa

Iowa City, IA
Alan B. Retik, M.D.

Harvard Medical School

Boston, MA

Anthony Schaeffer, M.D.

Northwestern University

Chicago, IL

Debra Slade, M.A.

Ridgefield, CT

Ann Stapleton, M.D.

University of Washington

Seattle, WA

William D. Steers, M.D.

University of Virginia

Charlottesville, VA

Leroy M. Nyberg, M.D., Ph.D.

NIH Consultant

Bethesda, MD 


\section{CONTENTS}

\section{Oncology and Cellular Biology}

1. Recent Developments in the Treatment of Bladder Cancer .............. 3 Raymond S. Lance and H. Barton Grossman

2. Interferon- $\alpha$ Response and Signal Transduction Pathway in Transitional Carcinoma Cell Lines

Sudip K. Bandyopadhyay, Raymond R. Rackley, Surena F. Matin, and Provash C. Sadhukhan

3. Molecular Cloning and Expression of Uroplakins in Transitional Cell

Carcinoma.....

Takeshi Yuasa, Tatsuhiro Yoshiki, Takahiro Isono, Tsutomu Tanaka, and Yusaku Okada

4. Application of a Novel Protein Chip Mass Spectrometry Technology for the Identification of Bladder Cancer-Associated Biomarkers

Antonia Vlahou, Paul F. Schellhammer, and George L. Wright, Jr.

5. The Photodynamic Diagnosis (PDD) for Early Detection of Carcinoma and Dysplasia of the Bladder....

A. Zumbraegel, K.-H. Bichler, F. S. Krause, G. Feil, and H. J. Nelde

6. Prediction of Lymph Node Metastasis Based on P53 and nm23-H1 Expression in Muscle Invasive Grade III Transitional Cell Carcinoma of Bladder.

Dong Soo Park, Young Tae Lee, and Jin Moo Lee

7. Detection of HER-2/neuCEP 17 Mutations at Invasive Bladder Cancer

Gerhard Feil, F. Steffen Krause, Andreas Zumbraegel, and Karl-H.

Bichler

8. Ki67, P53, nm23, and DNA Cytometry in Bladder Cancer: Potential

Markers for Detection of Recurrence?

Gerhard Feil, F. Steffen Krause, Andreas Zumbraegel, Hans W.

Wechsel, and Karl-H. Bichler

9. Comparison of Cytology and Nuclear Matrix Protein 22 (NMP 22) for the Detection and Follow-Up of Bladder Cancer 
Sven Lahme, Karl-Horst Bichler, Gerhard Feil, Andreas Zumbraegel, and Tobias Götz

10. The Effect of Contrast Material on Transitional Cell Carcinoma Viability Martha K. Terris

1.1. Fluorodeoxyglucose Positron Emission Tomography Studies in the Diagnosis and Staging of Transitional Cell Carcinoma

I. Jenna Liu, George M. Segall, Matilde Noni-Murcia, and Martha K. Terris

12. Radiochemotherapy in Locally Invasive Non-Metastatic Carcinoma of the Bladder.

J.L. Brod, R. Kühn, W. Schafhauser, and K.M. Schrott

13. Gene and Antisense Therapy of Bladder Cancer

Peter Ardelt, Ingo Kausch, and Andreas Böhle

14. Tumor Suppressor Genes of Bladder Cancer and Potential for Gene Therapy

Motoyoshi Tanaka and H. Barton Grossman

\section{Muscle, Matrix, and Obstruction}

15. Molecular Response of the Bladder to Obstruction. 195 Andrew J. Kirsch, Edward J. Macarak, Brahim Chaqour, Theresa Quinn, Stephen Zderic, Alan Flake, Timothy Crombleholme, N. Scott Adzick, Douglas Canning, Ann M. Hubbard, Joel Rosenbloom, Howard M. Snyder, and Pamela S. Howard

16. The Role of Lipids and Lipid Metabolites in Urinary Bladder Dysfunction Induced by Partial Outlet Obstruction.

Martha A. Hass and Robert M. Levin

17. Intercellular Communication and Bladder Function 239

George J. Christ, Karicheti Venkateswarlu, Nancy S. Day, Mira Valcic, Cristian Santizo, Weixin Zhao, Hong-Zhan Wang, Katerina Persson, and Karl-Erik Andersson

18. Ischemia as an Etiological Factor in Bladder Instability: Implications for Therapy

Alison F. Brading and Stephanie Symes 
19. Effect of Chronic Ischemia on Bladder Structure and Function 271 Kazem M. Azadzoi

20. Ultrastructural Diagnosis of Neuropathic Detrusor Overactivity: Validation of a Common Myogenic Mechanism

A. Haferkamp, J Dörsam, and A. Elbadawi

21. Serial Changes of Smooth Muscle Cell Phenotypes in Rat Urinary Bladder Following Partial Outflow Obstruction.

T. Hanai, S. Matsumoto, N. Ohnishi, and T. Kurita

22. Study of Detrusor Dysfunction Due to Outlet Obstruction: Link Between Analysis of Uroflows of Men with Benign Prostatic Hyperplasia and Animal Studies

Françoise A. Valentini, Robert M. Levin, Gilbert R. Besson, and Pierre P. Nelson

23. Ultrasonic Measurement of Bladder Weight as a Novel Urodynamic Modality

Osamu Ukimura, Munekado Kojima, Tsuyoshi Iwata, Mitsushiko Inaba, and Tsuneharu Miki

\section{Neurophysiology, Neurogenic Bladder, and Incontinence}

24. Experimental Neurogenic Cystitis 319 Luc Jasmin and Gabriella Janni

25. Murine In Vitro Whole Bladder Physiology 337 Joel C. Hutcheson, Raimund Stein, Douglas A. Canning, Alan J. Wein, Samuel K. Chacko, and Stephen A. Zderic

26. $\mathrm{Ca}^{2+}$ Sparks and $\mathrm{K}_{\mathrm{Ca}}$ Channels: Novel Mechanisms to Relax Urinary Bladder Smooth Muscle

Thomas J. Heppner, Gerald M. Herrera, Adrian D. Bonev, David HillEubanks, and Mark T. Nelson

27. The Effect of Intravesical Capsaicin and Resiniferatoxin in Neurogenic Bladder Dysfunction 359 Ahmed S. El-Mahrouky, Osama M. Elashry, and Mahmoud A. Emran

28. On the Physioanatomy of Micturition and Urinary Continence--New Concepts. 381 Ahmed Shafik 
29. Stress Urinary Incontinence: New Concept of Pathogenesis and Treatment by Pudendal Canal Decompression

Ahmed Shafik

30. Transcutaneous Electrovesicogram in Normal Volunteers and Patients with Interstitial Cystitis, Neurogenic Bladder, Benign Prostatic Hyperplasia, and after Cystectomy Ahmed Shafik

31. Extracorporeal Electromagnetic Stimulation for Urinary Incontinence and Bladder Disease

Roger P. Goldberg and Peter K. Sand

32. Pubovaginal Sling Surgery without Using Abdominal Leak Point Pressure: An Outcomes Analysis Jong M. Choe

33. The Use of Synthetic Materials in Pubovaginal Sling 481 Jong M. Choe

34. Surgical Implantation of the Synthetic Sling (The 6-Point Fixation Technique and Weight-Adjusted Spacing Nomogram): Technique and Results 493 Jong M. Choe

35. Reconstructive Surgery in Neuropathic Bladder 509 Bulent Cetinel

36. The Role of the Simple Cystectomy in Urologic Surgery 535 Endre Neulander, Inoel Rivera, and Zev Wajsman

\section{Immunology, Inflammation, and Infection}

37. Neurogenic Inflammation of the Bladder 551 Dale E. Bjorling, Matt Beckman, and Ricardo Saban

38. Urine-Induced Apoptosis in Cultured Bladder Urothelial Cells 585 Paul C. Stein, Jian Zhang, and C. Lowell Parsons

39. Bacillus of Calmette and Guerin Modulates Nuclear Factor Kappa $\beta$ in Two Urothelial Carcinoma Cell Lines 599 Louis S. Liou, Provash C. Sadhukhan, Sudip K. Bandyopadhyay, and Raymond R. Rackley 
40. Induction of Urothelial Cell Proliferation by Fibroblast Growth Factor-7. in RAG1-Deficient Mice 623 James A. Bassuk, Kimberly Cochrane, and Michael E. Mitchell

41. Development of a Recombinant FimCH Vaccine for Urinary Tract Infections 635 Solomon Langermann and W. Ripley Ballou

42. Intravesicular Pain Mapping Larry A. Demco and Marelyn Medina

43. A Hypothesis for the Etiology of Interstitial Cystitis 683 Ada Elgavish

44. Pentosanpolysulfate (Elmiron) is a Potent Inhibitor of Mast Cell Histamine Secretion

George Chiang, Phani Patra, Richard Letourneau, Sheila Jeudy, William Boucher, Marlon Green, Granum R. Sant, and Theoharis C. Theoharides

45. The Efficacy of Intravesicular Sterile Sodium Chondroitin Sulfate $0.2 \%$ in Potassium Tested Positive Patients with Interstitial Cystitis. 731 G. Steinhoff, B. Ittah, and S. Rowan

46. Cystectomy Causes Immunosuppression in Bladder Cancer .... 741 Ralf Herwig, Olaf A. Brinkmann, Karl-Dietrich Sievert, G. Brodner, and Lothar Hertle

\section{New Frontiers and Therapies}

47. Nocturia 751 Jeffrey P. Weiss and Jerry G. Blaivas

48. The Fate of Urinary Bladder Smooth Muscle after Outlet Obstruction

-A Role for the Sarcoplasmic Reticulum 773 Raimund Stein, ChaoLiang Gong, Joel Hutcheson, Lev Krasnopolsky, Douglas A. Canning, Michael Carr, and Stephen A. Zderic

49. Alteration of Collagen Three-Dimensional Architecture in Noncompliant Human Urinary Bladder 791

Karyn S. Eilber, Cortino Sukotjo, Shlomo Raz, and Ichiro Nishimura 
50. Modulation of Angiogenesis: Experimental Strategies and Potential Therapeutic Applications

Patricia G. Phillips, Linda M. Birnby, Amithi Narendran, and Wendy

L. Milonovich

51. Methods of Testing Urethral Resistance in the Female Rat

Margot S. Damaser, Fernando J. Kim, and Gina M. Minetti

52. Virtual Reality Surgical Simulation for Lower Urinary Tract

Endourologic Surgery

Michael J. Manyak, Kristin Santangelo, James Hahn, Roger Kaufman,

Thurston Carleton, Xing Cheng Hua, and Raymond J.Walsh

53. Autologous Muscle Transfer for Reconstruction of the Lower Urinary Tract

Arnulf Stenzl and Milomir Ninkovic

54. Effectiveness of Denuding the Intestinal Mucosa by Submucosal Injection in the Porcine Model

I. Jenna Liu and Martha K. Terris

55. Urinary Diversion in the Management of Chronic Perineal Skin Ulceration in Spinal Cord Injured Patients Following Sphincterotomy

Moritz Hansen and Martha K. Terris

56. The Artificial Urinary Bladder 885

Dorothea Rohrmann

57. Comparison of the Breaking Strength of Polyglactin Mesh in Urine, Serum, and Cell Culture Media

Charles D. Best and Martha K. Terris

58. Growth of Cultured Human Urothelial Cells Into Stratified Urothelial Sheet Suitable for Autografts 907

Yuan Yuan Zhang and Peter Frey

59. Future Perspectives in Bladder Reconstruction 921 Anthony Atala

Subject Index 